\title{
Article \\ Observation of Ultrashort Laser Pulse Evolution in a Silicon Photonic Crystal Waveguide
}

\author{
Xiaochun Wang ${ }^{1}{ }^{\oplus}$, Jiali Liao ${ }^{2}$, Jinghan Pan ${ }^{1}$, Heng Yang ${ }^{3}$ and Xiujian Li ${ }^{1, *}$ \\ 1 Department of Physics, College of Liberal Arts and Sciences, National University of Defense Technology, \\ Changsha 410073, China; xiaochunwang01@163.com (X.W.); panjinghan18@nudt.edu.cn (J.P.) \\ 2 School of Physics and Optoelectronic Engineering, Xidian University, Xi'an 710071, China; \\ liaojiali@xidian.edu.cn \\ 3 College of Information and Communication, National University of Defense Technology, \\ Changsha 410073, China; hengy@yeah.net \\ * Correspondence: xjli@nudt.edu.cn
}

Citation: Wang, X.; Liao, J.; Pan, J.; Yang, H.; Li, X. Observation of

Ultrashort Laser Pulse Evolution in a Silicon Photonic Crystal Waveguide. Micromachines 2021, 12, 911. https:// doi.org/10.3390/mi12080911

Academic Editors: Minhao Pu and Jing $\mathrm{Xu}$

Received: 19 June 2021

Accepted: 28 July 2021

Published: 30 July 2021

Publisher's Note: MDPI stays neutral with regard to jurisdictional claims in published maps and institutional affiliations.

Copyright: (c) 2021 by the authors. Licensee MDPI, Basel, Switzerland. This article is an open access article distributed under the terms and conditions of the Creative Commons Attribution (CC BY) license (https:// creativecommons.org/licenses/by/ $4.0 /)$.

\begin{abstract}
Using the sum frequency generation cross-correlation frequency-resolved optical gating (SFG-XFROG) measurement setup, we observed the soliton evolution of low energy pulse in an Si photonic crystal waveguide, and it exhibited the pulse broadening, blue shift, and evident pulse acceleration. The soliton evolution was also investigated by nonlinear Schrödinger equation (NLSE) modelling simulation, and the simulated results agreed well with the experimental measurements. The effects of waveguide length on the pulse evolution were analyzed; the results showed that the pulse width changed periodically with increasing waveguide length. The results further the understanding of the ultra-fast nonlinear dynamics of solitons in silicon waveguides, and are helpful to soliton-based functional elements on CMOS-compatible platforms.
\end{abstract}

Keywords: photonic crystal waveguide; pulse acceleration; blue shift

\section{Introduction}

With the capability to tightly confine optical modes [1], the compatibility with mature CMOS processes [2], and the extremely large nonlinearities enhanced by slow-light effects, the Si photonic crystal waveguides (PhCWs) have been attracting more and more intention. Four-wave-mixing, temporal soliton, optical filter, all-optical modulation, all-optical switching, ultralow-power all-optical signal processing and all-optical wavelength conversion have been demonstrated in Si PhCWs [1,3]. Basically, pulse propagation is affected by the nonlinear properties and the inherent dispersion of the Si PhCWs, and they can make the pulse propagation exhibit optical solitons effects at the anomalous dispersion region [4], which will be important for numerous applications of optical information processing [5-8]. Recently, the linear and nonlinear effects such as the group velocity dispersion (GVD), the self-phase modulation (SPM), two photon absorption (TPA), cross phase modulation (XPM), have been widely studied [9-13]. As the solitons are the nonlinear waves that exhibit invariant or recurrent behavior as they propagate through some materials [14,15], measuring and governing its propagation by the precise control of the dispersion and nonlinear effects is very helpful for many applications [16,17].

Actually, the FROG and SFG-XFROG are efficient tools for check the measurements of ultra-short laser pulses [18-20]. However, up to now, although we had checked the soliton behavior in the Si nanowire and PhCWs [21-26], there is unclarity about all details of the soliton propagation, especially the evolution dynamics of the low-energy soliton in the Si $\mathrm{PhCWs}$, which are considered to be suitable to manage the solitons for the Si nanowire [27].

Herein, based on a sensitive an SFG-XFROG ultra-short laser pulse measurement setup, we observed the picosecond pulse evolution, which shows the pulses blue shift and pulse shaping in Si PhCWs with low input pulse energy of several pico-joule. The pulse 
evolution has also been investigated by the optimized nonlinear Schrödinger equation (NLSE) modeling simulation, and the NLSE predicted results agree well the experimental measurements. The research results provide valuable information for the design of waveguides [28] for various on-chip photonic circuits, in support of the ultra-broadband high-speed optical communications and optical signal processing.

\section{Measurement Setup and Observations of Pulse Evolution}

The SFG-XFROG experimental measurement setup for the ultrashort pulse characterization is shown in Figure 1, and it can provide sensitive and accurate measurement of the optical pulses output from the silicon photonic crystal waveguides in the time and spectral domains. A BBO crystal (1 mm thickness, provided by CASTECH INC. CHINA) and a Horiba JY FHR1000 spectrometer with a SYMPHONY II UVCCD-1024 $\times 256$-BIDD detector were applied to enable the minimum detected pulse energy to be as low as 500 attojoule (aJ), the spectral resolution to be better than $0.1 \mathrm{~nm}$, and the time resolution to be less than 1 femtosecond (fs). The measurement setup provides the detailed properties of the ultrashort pulses, including the time and frequency intensity and phase distributions, which help to capture the soliton dynamics. The Si PhCWs used in this work are the same as Ref. [26].

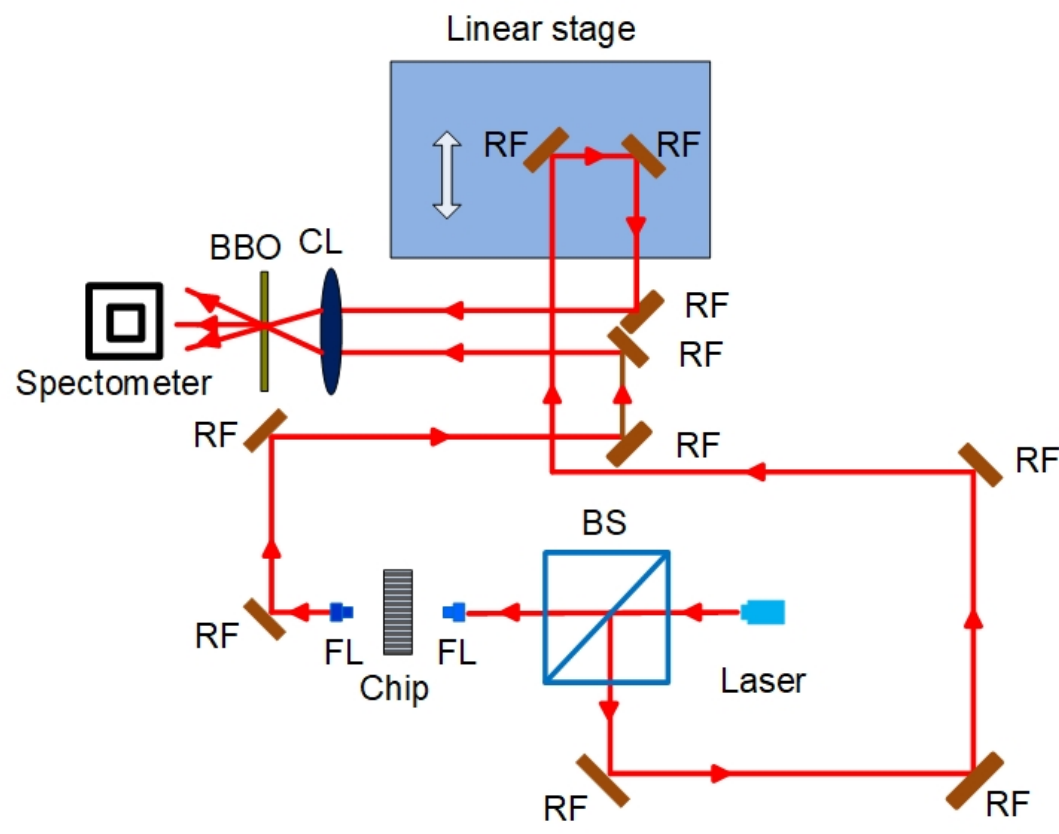

Figure 1. Experimental measurement setup of SFG-XFROG (RF: Reflector, BS: Beam splitter, FL: Focusing lens, CL: Bi-convex lens, BBO: Barium borate crystal).

Utilizing the SFG-FROG, we have measured the input pulse, and the measured trace of the input pulse is shown in Figure 2a; the temporal and spectral intensity profiles are extracted from this trace, and shown in the bottom of Figure 2c,d. The FROG retrieving error is as low as $0.075 \%$ for the reconstruction processes. The pulse duration and central wavelength of the input pulse are $2.25 \mathrm{ps}$ and $1555 \mathrm{~nm}$, respectively. The output pulses from the $\mathrm{Si} \mathrm{PhCWs}$ with increasing input pulse energies $E_{p}$ have been characterized using the SFG-XFROG, and the measured trace at $E_{p}=22 \mathrm{pJ}$ is shown in Figure 2b, where the XFROG retrieving error is $0.13 \%$.

The experimental results demonstrate that the output pulse is broadened after passing the waveguide as shown in Figure 2c. The broadening effect of the output pulse weakens as the FWHM of the output pulse shrinks from $3.91 \mathrm{ps}$ to $2.50 \mathrm{ps}$, corresponding to a broadening factor $\left(F W H M_{\text {output }} / F W H M_{\text {input }}\right)$ from 1.74 to 1.11 , with the pulse energy increasing from $59.5 \mathrm{fJ}$ to $13.1 \mathrm{pJ}$. However, at the pulse energy of $22.0 \mathrm{pJ}$, the pulse exhibited slight compression with the compression factor $\left(F W H M_{\text {output }} / F W H M_{\text {input }}\right)$ of 
0.98. The temporal domain profile shows that the FWHM of the output pulse is affected by the input pulse energy. The FWHM of the output pulse was broadening first and then compressing with the increasing of input pulse energy. The output pulse shows obvious pulse acceleration. The largest pulse acceleration is $2.17 \mathrm{ps}$ with $22.0 \mathrm{pJ}$ input energy. The output pulse is still smooth and not destroyed in the experiments.

The Figure $2 \mathrm{~d}$ shows the normalized intensity profiles of the output pulses in spectral domain. The pulse shows red shift with the low input pulse energy and blue shift with the high input pulse energy in Figure 2d. The largest red shift is $0.41 \mathrm{~nm}$ with an input energy of $59.5 \mathrm{fJ}$, and the largest blue shift is $0.68 \mathrm{~nm}$ with an input energy of $22.0 \mathrm{pJ}$.

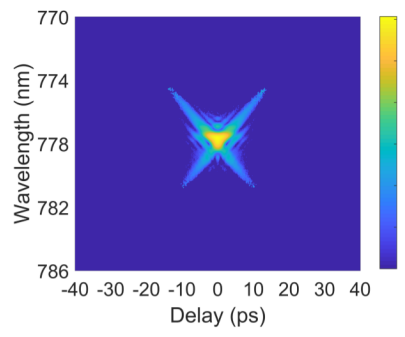

(a)

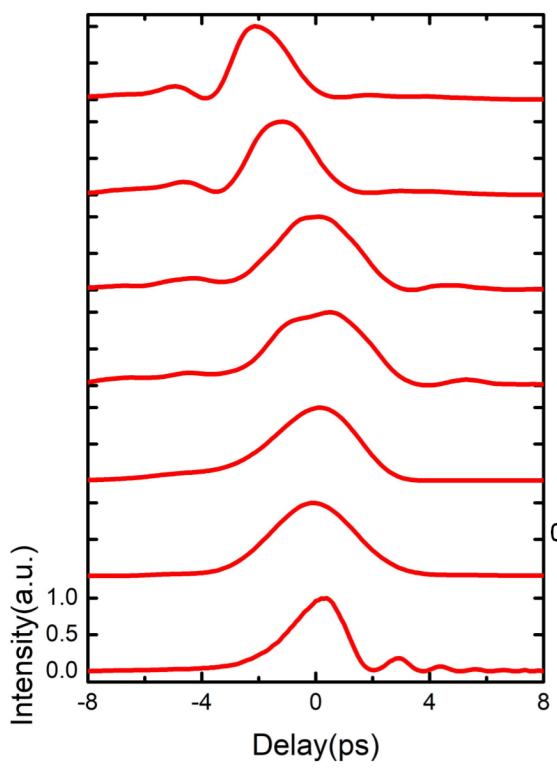

(c)

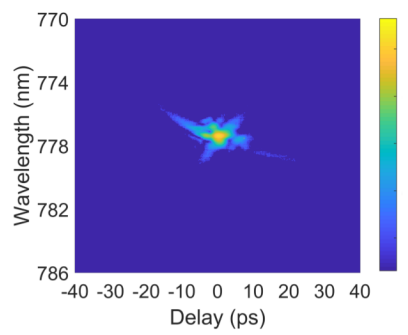

(b)

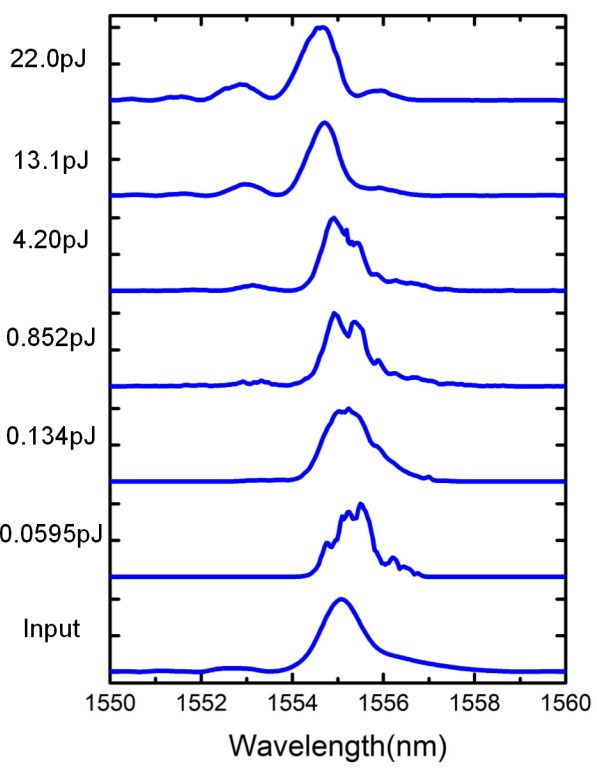

(d)

Figure 2. The output SFG-FROG/XFROG measurements, (a) FROG trace of the input pulse, (b) XFROG trace of the output pulse corresponding to Ep $=22.0 \mathrm{pJ}$, (c) the normalized intensity profiles in temporal domain, (d) the normalized intensity profiles in the spectral domain.

\section{Optimized NLSE Modeling Simulations and Discussions}

In order to unveil the principles of the pulse broadening and spectrum shift as shown in Figure 2, we perform the optimized nonlinear Schrödinger equation (NLSE) modeling simulations [26].

$$
\begin{gathered}
\frac{\partial A}{\partial z}+i \frac{\beta_{2}}{2} \frac{\partial^{2} A}{\partial^{2} t}-\frac{\alpha_{e f f}}{2} A=i\left(\gamma_{e f f}-\alpha_{T P A . e f f}\right)|A|^{2} A+\left(i k_{0} k_{c . e f f}-\frac{\sigma_{e f f}}{2}\right) N_{c} A \\
\frac{\partial N_{c}(z, t)}{\partial t}=\frac{\alpha_{T P A . e f f}}{2 h v_{0}}|A(z, t)|^{4}-\frac{N_{c}(z, t)}{\tau_{c}}
\end{gathered}
$$

where the group velocity dispersion (GVD) $\beta_{2}=-1800 \mathrm{ps}^{2} / \mathrm{m}$; The Kerr coefficient is $1.02 \times 10^{3}(1 \mathrm{~W} / \mathrm{m})$; The effective TPA coefficients is $13.4 \times 10^{-12} \mathrm{~m} / \mathrm{W}$; The effective free carrier dispersion and absorption parameters are $k_{c . e f f}=-13.3 \times 10^{-27} \mathrm{~m}^{3}$ and 
$\sigma_{e f f}=4.62 \times 10^{-21} \mathrm{~m}^{2}$, respectively. $A$ is the slow varying envelope of the propagation pulse electric amplitude; $N_{c}$ is the density of free carrier; $Z$ is the pulse transmission position inside the waveguide. $\tau_{c}$ is the free carrier effective lifetime, which is estimated to $0.5 \times 10^{-9} \mathrm{~s}$. $h$ and $v_{0}$ are the Plank constant and light frequency, respectively [26].

Figure 3 shows the experimental and simulation results for $1555 \mathrm{~nm}$ with the input pulse energy of $0.134 \mathrm{pJ}$ and $22.0 \mathrm{pJ}$, respectively. In the simulations, the input pulse profile measured by SFG-FROG is used as the initial pulse. Obviously, the major experimental results are matched with the NLSE simulation results, which indicates that the optimized NLSE modeling and the parameters are suitable for the Si PhCWs, which illustrate the NLSE modeling simulation can properly predict pulse evolution dynamics in the Si PhCWs. However, we can also find that, some slight periodic oscillation side wings appear around the main peak of the simulation results, which are induced by the FFT-window selection of the split-step solution method for the NLSE, and can be considered noise perturbations of the simulation.

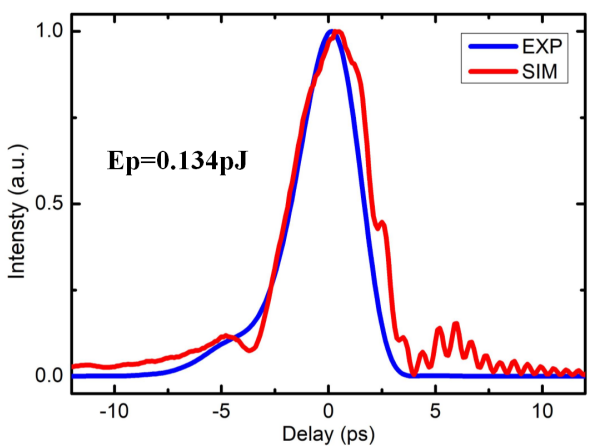

(a)

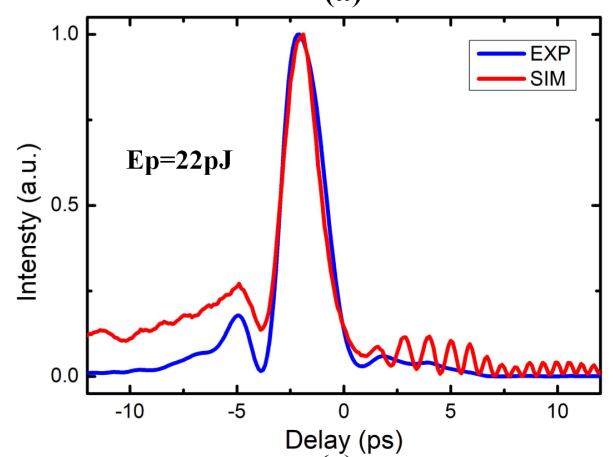

(c)

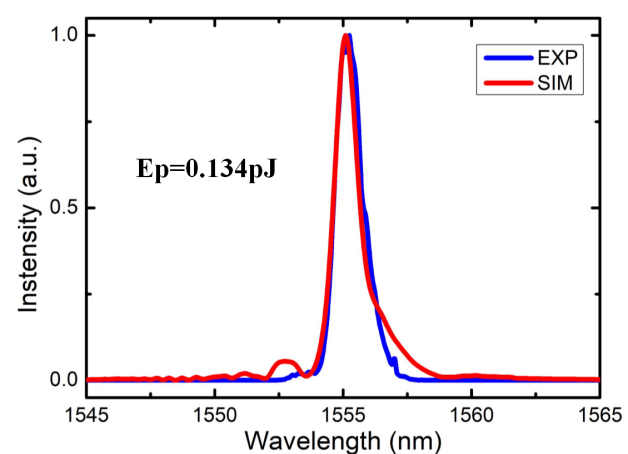

(b)



(d)

Figure 3. The experimental and the optimized NLSE simulation results for various pulse energies, $(\mathbf{a}, \mathbf{c})$ temporal profiles , (b,d) spectral profiles of the pulse, $(\mathbf{a}, \mathbf{b})$ with $0.134 \mathrm{pJ}$ input pulse energy, (c,d) with $22.0 \mathrm{pJ}$ input pulse energy. The blue solid line are experimental results, and the red solid line are optimized NLSE simulation results.

The input pulse for the simulations is the experiment input pulse at $22.0 \mathrm{pJ}$ with center wavelength $1555 \mathrm{~nm}$. By increasing the $\mathrm{Si} \mathrm{PhCW}$ length, we calculate the output pulse widths at different propagation distances, and the results are shown in Figure 4. It is shown that the pulse temporal FWHM is transformed periodically with increasing propagation distance. In each period, the temporal FWHM is gradually compressed to the narrowest, and then sudden broadening occurs in the transmission following the maximum compression. Because of the large losses in the Si PhCWs, the minimum pulse width increases with the soliton evolution periods. The pulse transmission emerges the soliton with the balance between the nonlinear effects and the dispersion in the Si PhCWs. 


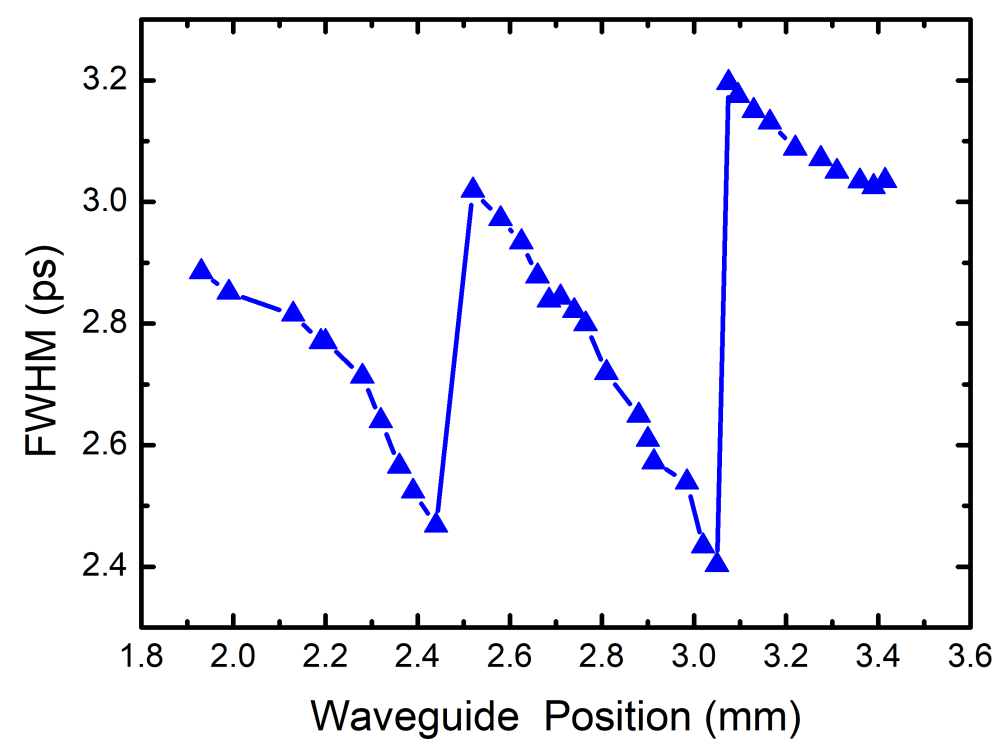

Figure 4. The FWHM of output pulse temporal profiles by the NLSE simulations with different waveguide position for $22.0 \mathrm{pJ}$ input pulse energy at $1555 \mathrm{~nm}$.

\section{Conclusions}

In summary, using sensitive SFG-XFROG measurements, we observed pulse broadening with a broaden factor of 1.74 , blue shift, red shift and obvious pulse acceleration when the temporal soliton transmitted through the Si PhCW at a center wavelength of $1555 \mathrm{~nm}$. The optimized NLSE simulation results matched the measurements. The results show that the FWHM of pulse gradually changes to the narrowest, and then broadening occurs following the narrowest point along the propagation distance, i.e., the simulation results show that periodic solitons are produced in the process of pulse transmission. Through the study, we can optimize the Si PhCWs for pulse shaping. These results help us understand how ultra-fast pulses propagate in silicon-based waveguides, and even open the way for soliton-based functional elements in CMOS-compatible platforms.

Author Contributions: Conceptualization , X.L. and X.W.; methodology, X.W.; software, J.L.; validation, X.W.; investigation, J.P. and X.W.; data curation, X.W.; writing, X.W., J.L., J.P., H.Y. and X.L. supervision, X.L. All authors have read and agreed to the published version of the manuscript.

Funding: This research was funded by the National Science Foundation of China (NSFC) (61070040, 61108089, 61205087, 61107005 62005207), Hunan Provincial Natural Science Foundation of China (805297133191), Natural Science Foundation of Shaanxi Province (2019JQ-648).

Conflicts of Interest: The authors declare no conflict of interest.

\section{References}

1. Almeida, V.R.; Barrios, C.A.; Panepucci, R.R.; Lipson, M. All-optical control of light on a silicon chip. Nature 2004, 431, 1081-1084. [CrossRef]

2. Dai, D.; Bowers, J.E. Novel concept for ultracompact polarization splitter-rotator based on silicon nanowires. Opt. Express 2011, 19, 10940-10949. [CrossRef] [PubMed]

3. Foster, M.A.; Turner, A.C.; Sharping, J.E.; Schmidt, B.S.; Lipson, M.; Gaeta, A.L. Broad-band optical parametric gain on a silicon photonic chip. Nature 2006, 441, 960-963. [CrossRef] [PubMed]

4. Agrawal, G.P. Chapter 2-Pulse Propagation in Fibers, in Nonlinear Fiber Optics, 4th ed.; Academic Press: San Diego, CA, USA, 2006.

5. Koch, J.; Li, S.; Pachnicke, S. Transmission of Higher Order Solitons Created by Optical Multiplexing. J. Light. Technol. 2019, 37, 933-941. [CrossRef]

6. Sedov, E.V.; Redyuk, A.A.; Fedoruk, M.P.; Gelash, A.A.; Frumin, L.L.; Turitsyn, S.K. Soliton content in the standard optical OFDM signal. Opt. Lett. 2018, 43, 5985-5988. [CrossRef] [PubMed]

7. Marpaung, D.; Pagani, M.; Morrison, B.; Eggleton, B.J. Nonlinear Integrated Microwave Photonics. J. Light. Technol. 2014, 32, 3421-3427. [CrossRef] 
8. Zou, X.; Lu, B.; Pan, W.; Yan, L.; Stöhr, A.; Yao, J. Photonics for microwave measurements. Laser Photonics Rev. 2016, 10, 711-734. [CrossRef]

9. Nakahama, T.; Ozaki, N.; Oda, H.; Ikeda, N.; Sugimoto, Y. Numerical investigation of highly efficient and tunable terahertz-wave generation using a low-group-velocity and low-dispersion two-dimensional GaAs photonic crystal waveguide. Jpn. J. Appl. Phys. 2020, 59, 90903. [CrossRef]

10. Hsieh, I.; Chen, X.; Dadap, J.I.; Panoiu, N.C.; Osgood, R.M.; McNab, S.J.; Vlasov, Y.A. Ultrafast-pulse self-phase modulation and third-order dispersion in Si photonic wire-waveguides. Opt. Express 2006, 14, 12380-12387. [CrossRef]

11. Chaturvedi, D.; Mishra, A.K.; Kumar, A. Self-phase modulation-induced modulation instability in silicon-on-insulator nanowaveguides. Opt. Laser Technol. 2019, 119, 105578. [CrossRef]

12. Pu, M.; Hu, H.; Ottaviano, L.; Semenova, E.; Vukovic, D.; Oxenløwe, L.K.; Yvind, K. Ultra-Efficient and Broadband Nonlinear AlGaAs-on-Insulator Chip for Low-Power Optical Signal Processing. Laser Photonics Rev. 2018, 12, 1800111. [CrossRef]

13. Ooi, K.J.A.; Ng, D.K.T.; Wang, T.; Chee, A.K.L.; Ng, S.K.; Wang, Q.; Ang, L.K.; Agarwal, A.M.; Kimerling, L.C.; et al. Pushing the limits of CMOS optical parametric amplifiers with USRN:Si $\mathrm{N}_{3}$ above the two-photon absorption edge. Nat. Commun. 2017, 8, 13878. [CrossRef]

14. Colman, P.; Husko, C.; Combrié, S.; Sagnes, I.; Wong, C.W.; Rossi, A.D. Temporal solitons and pulse compression in photonic crystal waveguides. Nat. Photonics 2010, 4, 862-868. [CrossRef]

15. Blanco-Redondo, A.; Husko, C.; Eades, D.; Zhang, Y.; Li, J.; Krauss, T.F.; Eggleton, B.J. Observation of soliton compression in silicon photonic crystals. Nat. Commun. 2014, 5, 3160. [CrossRef]

16. Liu, J.; Raja, A.S.; Karpov, M.; Ghadiani, B.; Pfeiffer, M.H.P.; Du, B.; Engelsen, N.J.; Guo, H.; Zervas, M.; Kippenberg, T.J. Ultralow-power chip-based soliton microcombs for photonic integration. Optica 2018, 5, 1347-1353. [CrossRef]

17. Soysouvanh, S.; Jalil, M.A.; Amiri, I.S.; Ali, J.; Singh, G.; Mitatha, S.; Yupapin, P.; Grattan, K.T.V.; Yoshida, M. Ultra-fast electrooptic switching control using a soliton pulse within a modified add-drop multiplexer. Microsyst. Technol. 2018, 24, 3777-3782. [CrossRef]

18. Efimov, A.; Taylor, A.J. Cross-correlation frequency-resolved optical gating for studying ultrashort-pulse nonlinear dynamics in arbitrary fibers. Appl. Opt. 2005, 44, 4408-4411. [CrossRef] [PubMed]

19. Dudley, J.M.; Gu, X.; Xu, L.; Kimmel, M.; Zeek, E.; Shea, P.O.; Trebino, R.; Coen, S.; Windeler, R.S. Cross-correlation frequency resolved optical gating analysis of broadband continuum generation in photonic crystal fiber: simulations and experiments. Opt. Express 2002, 10, 1215-1221. [CrossRef]

20. Tsurumachi, N.; Hikosaka, K.; Wang, X.; Ogura, M.; Watanabe, N.; Hattori, T. Observation of ultrashort pulse propagation anisotropy in a semiconductor quantum nanostructure optical waveguide by cross-correlation frequency resolved optical gating spectroscopy. Appl. Phys. 2003, 94, 2616-2621. [CrossRef]

21. Marko, M.; Veitia, A.; Li, X.; Zheng, J. Disturbance of soliton pulse propagation from higher-order dispersive waveguides. Appl. Opt. 2013, 52, 4813-4819. [CrossRef] [PubMed]

22. Liao, J.; Marko, M.; Li, X.; Jia, H.; Liu, J.; Tan, Y.; Yang, J.; Zhang, Y.; Tang, W.; Yu, M.; Lo, G.; Kwong, D.; Wong, C.W. Crosscorrelation frequency-resolved optical gating and dynamics of temporal solitons in silicon nanowire waveguides. Opt. Lett. 2013, 38, 4401-4404. [CrossRef] [PubMed]

23. Marko, M.D.; Li, X.; Zheng, J.; Liao, J.; Yu, M.; Lo, G.; Kwong, D.; Husko, C.A.; Wong, C.W. Phase-resolved observations of optical pulse propagation in chip-scale silicon nanowires. Appl. Phys. Lett. 2013, 103, 21103. [CrossRef]

24. Marko, M.; Li, X.; Zheng, J. Soliton propagation with cross-phase modulation in silicon photonic crystal waveguides. J. Opt. Soc. Am. B 2013, 30, 2100-2106. [CrossRef]

25. Liu, K.; Zhang, J.F.; Xu, W.; Zhu, Z.H.; Guo, C.C.; Li, X.J.; Qin, S.Q. Ultra-fast pulse propagation in nonlinear graphene/silicon ridge waveguide. Sci. Rep. UK 2015, 5, 16734. [CrossRef] [PubMed]

26. Li, X.; Liao, J.; Nie, Y.; Marko, M.; Jia, H.; Liu, J.; Wang, X.; Wong, C.W. Unambiguous demonstration of soliton evolution in slow-light silicon photonic crystal waveguides with SFG-XFROG. Opt. Express 2015, 23, 10282-10292. [CrossRef] [PubMed]

27. Zhang, J.; Lin, Q.; Piredda, G.; Boyd, R.W.; Agrawal, G.P.; Fauchet, P.M. Optical solitons in a silicon waveguide. Opt. Express 2007, 15, 7682-7688. [CrossRef] [PubMed]

28. Fu, M.; Liao, J.; Shao, Z.; Marko, M.; Zhang, Y.; Wang, X.; Li, X. Finely engineered slow light photonic crystal waveguides for efficient wideband wavelength-independent higher-order temporal solitons. Appl. Opt. 2016, 55, 3740-3745. [CrossRef] 